\title{
Subject Index Vol. 40, 1996
}

Acylated carnitine 221 Adipose tissue, pig 287 Age 351 Altitude 315 Amino acids 10

Anthropometry 123

Basic red cell ferritin 331 Biochemical parameters 146 Blood lipid(s) 71

- levels 116

pressure, rat 1

urea 359

Body composition, children 123

impedance 123

weight gain 165 Brush border enzyme 269

Ca metabolism 81 Caecum 283 Calorimetry 10 Carbohydrate(s) 116,336

- ligands 252

L-Carnitine 221

Cell adhesion molecules 252 Children 331 Cholecystokinin 109 Cholesterol secretion 157

Colonic damage 175,227 Creatine administration 359 Crohn's disease 137,331

Dairy fat 287 Dexfenfluramine 10 Diet 315,343 Dietary fats 269

- $\quad$ intake 24

- $\quad$ survey, South German popula

tion 129

Docosahexaenoic acid 91, 234,263 Dual-energy X-ray absorp-tiometry 123

Early gene expression 303 Egg 109

Electroretinogram 91 Emeriamine 157 Energy balance 165

- $\quad$ intake 146

metabolism 10

partitioning 165 Energy-restricted dietary regimen

212 Enterostatin 212 Essential fatty acids 234

- - n-3 91,175,227

- - - andn-6 325 Ethanol 277,283 Exercise 359

Fatty acid(s) composition 175, 227

- deficiency 91

- oxidation inhibitor 157

-, $\omega-361,303$

-, $\omega-6303$ Fe deficiency 81

metabolism 81

Fetal development, costs 165

Fiber 343

Fish oil 61

Focusing 296

Folic acid absorption 277,283 
Food consumption, trends 129

efficiency 165

frequency questionnaire 129

intake 146

restriction 165 Free fatty acids 10 Frequently sampled intravenous

glucose tolerance test 116

Gangliosides, human milk 194 Gas exchange 336 Gastric emptying 109

inhibitory peptide 109 Gene expression 303 Glucose 10

response 109

tolerance 61 Glutathione peroxidase 296 Glycogen 10

Glycolysis 303 Glycosylation 269 Goblet cell 343 Guinea pig 91

367

Haematological parameters 146 Hairless mice 52 Hay's diet 336 Heat production 336 Human milk 252 Hyperinsulinemic-euglycemic clamp 116

Infant formula fat blends 234

- $\quad$ nutrition 252

Inflammation 303

Inflammatory bowel disease 175 ,

227,331 Insulin 10,212

secretion 116

sensitivity 116 Iron status 331

Jejunum 277

Juvenile chronic arthritis 137

Lactation 194,221 Lactobacillus 137 Leukotriene B4 $175 \alpha$-Linolenic acid 175, $227 \gamma$-Linolenic acid 99 Lipids 269 Lipogenesis 287, 303 Liver 287

- $\quad$ transglutaminases 52

Longissimus dorsi muscle 287

Low-protein diet 1

Lymphocyte proliferation 71

Mammals 221 Mediterranean diet 202 Methyltetrahydrofolate absorption 277, $283 \mathrm{Mg}$

metabolism 81 Milk 221

- $\quad$ formulas 221

Monounsaturated fatty acids 116

Mucosa 343

Mucus 343

Muscle glycogen 359

Natural killer cells 71 Nutrient biochemical assessment 24

- $\quad$ intake 146

Nutrition survey 146

Oat-based liquid food 212 Obesity 10 Oleic acid 71

Olive oil 71 ß-Oxidation 221

Palm oil 263 Panamanian mothers, milk

gangliosides 194 Perfused rat liver 157 Perilla oil emulsion 175, 227 Phospholipid 99 Physical exercise 202 Plasma 296

$\operatorname{lipid}(\mathrm{s}) 202$

- levels 212 Polyunsaturated fatty acids 116 Pregnancy 165 
Premature infant 325 Prostaglandin 99 Protein 336 Pyruvate kinase 10

Rat(s) 351

- $\quad$ intestine 269

Refeeding 351

Salt 1

Sardine oil 263 Saturated fatty acids 116 Saturated/unsaturated fatty acids 269 Schoolchildren, breakfast 146 Selectins 252 Selenium 296

- $\quad$ status 296

Selenoproteins 296

Small intestine 351

Sows 336

Spanish mothers, milk gangliosides 194 Spirulina 99 Starvation 351 Subcutaneous backfat 287

Thromboxane $99 \alpha$-Tocopherol 325 Total parenteral nutrition 227 Triglyceride secretion 157

Ultrastructure, jejunal absorptive

cells 263

Vegetarian diet 202 Vitamin(s) 24

A deficiency 52

E 325

Weight loss 212,315

Subject Index Vol. 40, 1996 